\title{
Investigating the Role of Mucin as Frontline Defense of Mucosal Surfaces against Mycobacterium avium Subsp. hominissuis
}

\author{
Jessica Bechler' and Luiz E. Bermudez $\mathbb{D D}^{1,2}$ \\ ${ }^{1}$ Department of Biomedical Sciences, Carlson College of Veterinary Medicine, 106 Dryden Hall Corvallis, Corvallis, \\ OR 97331, USA \\ ${ }^{2}$ Department of Microbiology, College of Science, Oregon State University, Corvallis, USA
}

Correspondence should be addressed to Luiz E. Bermudez; luiz.bermudez@oregonstate.edu

Received 7 May 2020; Accepted 11 June 2020; Published 1 July 2020

Academic Editor: Giovanna Franciosa

Copyright $\odot 2020$ Jessica Bechler and Luiz E. Bermudez. This is an open access article distributed under the Creative Commons Attribution License, which permits unrestricted use, distribution, and reproduction in any medium, provided the original work is properly cited.

\begin{abstract}
Mycobacterium avium is a human and animal pathogen that infects the host through the mucosal surfaces. Past work has demonstrated that the bacterium can interact with both the respiratory and gastrointestinal tracts. Those surfaces in the body are covered by a bilayer of a glycoprotein, mucin, which works as a physical barrier and a gel which contains antibacterial and antivirus properties. This current work shows that different strains of M. avium, in contrast to Escherichia coli, Pseudomonas aeruginosa, and Listeria monocytogenes, are not able to bind to mucins, MUC2 and MUC5b, the main mucins in the gastrointestinal and respiratory tracts, respectively. The lack of binding is due to the characteristics of the cell wall and is impaired by altering lipids, proteins, or glycolipids. M. avium, in contrast to E. coli, interacts with epithelial cells equally in the presence or absence of the mucin, suggesting that the cell wall of the pathogen can facilitate the bacterial movement through the mucin layer, towards the mucosal wall. In conclusion, the study has shown that M. avium can avoid the mucin barrier, which explains its ability to interact with the mucosal epithelium, even in absence of motion-related structures.
\end{abstract}

\section{Introduction}

Mycobacteriumavium subsp. hominissuis (M. avium) is an environmental bacteria associated with the infection of individuals with underlying lung conditions such as bronchiectasis, cystic fibrosis, and emphysema $[1,2]$. Infections caused by $M$. avium are also seen in patients with immunosuppression, such as AIDS and specific immunodeficiencies [3].

M.avium interacts with the host mucosal cells once in the airways or in the GI tract. Epithelial mucosal cells are invaded by the pathogen both in vitro [4] and in vivo [5] as a step toward the infection. M. avium pathogenesis of the lung is also associated with the formation of a biofilm on the surface of the mucosa $[6,7]$. This infection is complex and involves the formation of microaggregates once they bind to the epithelial mucosa and stimulation of cytoskeleton rearrangement, stages that require the participation of many bacterial proteins $[8,9]$.
Healthy airway surfaces are coated with a mucus layer that is largely composed of water, salts, mucins, and surfactants. As a step in the process of interaction with the airway or the GI mucosa, pathogens need to bypass the mucin layer, both in the respiratory tract as well as the intestinal tract. In the intestinal lumen, mucin is present in two layers, one adjacent to the mucosal surface, which is rich in antimicrobial peptides, and a more external layer, where bacteria are encountered $[10,11]$. The same arrangement appears to be present in the airways [12].

Mucins are high-molecular mass glycoproteins composed of a protein core with carbohydrate and sulfate molecules. Mucins have characteristic Pro-Thr-Ser repeats as well as a Cys-rich domain that provide key disulfide bonding capacity, important for mucin multimerization and mucus function. The major mucins produced in the airways are the secreted polymeric mucins MUC5AC and MUC5B [13], while in the GI mucosal surface, MUC2 is more abundant [11]. In mammalians, the mucin/mucus involves 
the coordinated activities of secretory cells that release polymeric mucin glycoproteins and ciliated cells that transports and eliminates foreign material including bacteria [13]. Bergstrom et al. and colleagues [14] showed that intestinal MUC2 can protect against lethal infectious colitis by disassociating pathogenic and commensal bacteria from the colonic mucosa. The authors showed that MUC2 production is critical for host protection during attachment and effacing bacterial infections (Escherichia coli and Citrobacter rodentium) but limits the overall bacterial pathogens and commensal numbers associated with the mucosal surface. Mucins also reduce surface adhesion and biofilm formation of Pseudomonas aeruginosa [15].

In addition, the mucin interacts with pulmonary macrophages (alveolar and submucosal) in several capacities in distinct anatomic locations. The viscosity of the environment caused by the glycoprotein mucin is very important to keep pathogens at some distance from the mucosal surface. For some pathogens, motility plays an important role in pathogenesis and is crucial to organisms such as Pseudomonas aeruginosa to colonize the host and form biofilms [16].

The hallmark phenotype of cystic fibrosis is dehydration of the mucosal layer across all the epithelial surfaces in the body.

The abilities of secreted mucins to regulate mucocellular clearances are dependent on their polymer structures formed through disulfide bonds. Pathogenic bacteria such as enterotoxic Escherichia coli produces a protein, EatA, that degrades the intestinal mucin [17], and therefore can migrate towards and ultimately bind to the mucosal surface.

Because the role of mucins has not been studied with regards to $M$. avium infection, we hypothesized that the mucin is probably ineffective in its role to separate mycobacteria from the mucosal layer of cells, likely due to the lipid content of the mycobacterial surface, therefore facilitating the bacterium movements toward the mucosal wall. The results of the present investigation support the hypothesis that the M. avium surface protects the bacterium against the action of mucins.

\section{Materials and Methods}

\subsection{Bacteria}

M. avium 104 was isolated from the blood of a patient (the characteristics of the bacteria have been published in [18]). Bacteria were cultured in the Middlebrook 7H10 agar medium (Difco Laboratories, Detroit, MI), supplemented with oleic acid, albumin, dextrose, and catalase (OADC). $M$. avium 3388 obtained from Barbara Brown (Taylor, Texas) was cultured in the Middlebrook 7H10 agar medium described above. It was a lung pathogen. Mycobacterium avium (MAH) A5 and MAH A5 GPL/4B2 mutant were previously described $[18,19]$.

Pseudomonas aeruginosa PD0300 mucA22 mucoid variant was kindly provided by Martin Schuster (Oregon State University). P. aeruginosa was grown in the Mueller-Hinton broth. Bacteria were washed $(3,000 \times \mathrm{g})$, and the inoculum was established in the phosphate buffer solution (PBS) and inoculated in the 7H9 broth. Escherichia coli ATCC CFT073, Staphylococcus aureus ATCC 25923, and Listeria monocytogenes (clinical isolate) were cultured on the Luria-Bertani (LB) agar.

Prior to performing the assays, dilutions were made in Hank's balanced salt solution (HBSS) to match McFarland standards. Bacteria were prepared at $10^{5}, 10^{6}$, and $10^{7}$ concentrations.

2.2. Mucin. Purified gastric mucin MUC2 and pulmonary MUC5b purchased from Pfaltz and Bauer Inc. were added to a $2 \%$ agar gel at $5 \% w / v$. Agar $2 \% w / v$ was used as the control. Twenty four well tissue culture plates were used with approximately $400 \mu \mathrm{L}$ in each well for the binding assays.

2.3. Binding to Mucin Assay. Bacteria inoculum prepared in HBSS at the $\mathrm{pH} 7.3$ was diluted to a concentration of approximately $6 \times 10^{3} \mathrm{CFU} / \mathrm{ml}$ as determined by comparison with the McFarland standard. Then, $200 \mu \mathrm{L}$ of the suspension was added to each tissue culture plate well. The plate containing the agar, agar plus mucin, and the final bacterial suspension was incubated for $3 \mathrm{~h}$ at $37^{\circ} \mathrm{C}$ under anaerobic conditions (anaerobic jar, BD BBL GasPak jar). After the incubation period, each well was washed three times with $300 \mu \mathrm{L}$ of HBSS, and the removed supernatant was collected in $5 \mathrm{~mL}$ plastic tubes. Then, it was diluted by $10^{-3}$, and the final suspensions were plated onto the 7H10 agar or LB agar plates. The number of CFU on the plates was determined after 10 days for mycobacteria and 24-48 hours for other bacteria (Figure 1).

2.4. Pretreatment of Bacteria. To evaluate a variety of environmental conditions, usually encountered in the process of infection that could impact $M$. avium binding to the mucin, bacteria were preincubated under different conditions, and the mucin attachment experiments were repeated. $M$. avium is encountered in diverse environmental conditions, such as water. In addition, $M$. avium before reaching the intestinal tract has to pass through the stomach $(2 \mathrm{~h}$ emptying time). The $\mathrm{pH}$ of the stomach was considered to be 3.4, following ingestion of food. After incubation under the environmental condition, bacteria were spun down to $4^{\circ} \mathrm{C}$, and the concentration of the bacteria on the suspension was determined by the turbidity [20].

To determine the role of lipids and proteins on the bacterial surface during binding, mycobacteria were incubated with either Tween-20 for $3 \mathrm{~h}$ at $37^{\circ} \mathrm{C}$ or $50 \%$ trypsin for $3 \mathrm{~h}$ at $37^{\circ} \mathrm{C}$. Following treatment, bacteria were spun down and the final inoculum was prepared at $4^{\circ} \mathrm{C}$ to prevent the change of the bacterial phenotype as described above.

To evaluate whether the bacterial surface was involved in the property of not binding to the mucin, we use the $4 \mathrm{~B} 2$ mutant, which contains a mutation in the gene $p s t B$ involved in GPL synthesis, a mutant that has an altered colony morphotype [19]. 


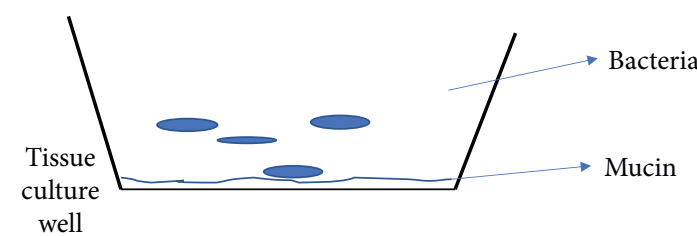

(a)

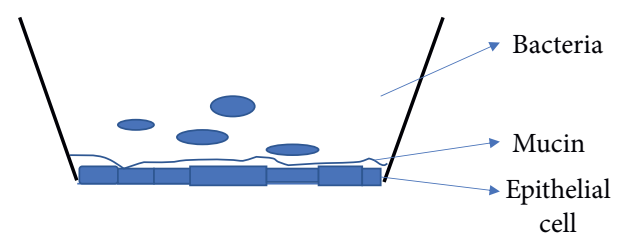

(b)

Figure 1: Models used to determine the binding of bacteria to the mucin and epithelial cell invasion: (a) polypropylene well and (b) epithelial cells covered with a layer of mucin.

2.5. Invasion Assay. Laryngeal cell line HEp-2, purchased from ATCC (American Tissue Culture Collection), was maintained in RPMI-1640 supplemented with $10 \%$ fetal bovine serum (FBS). Lung alveolar epithelial cells (A549) obtained from ATCC were cultured in DMEM supplemented with $10 \%$ FBS. Monolayers were prepared by seeding $10^{5}$ cells in each well of a 24 -well tissue culture plate and incubated until achieving $90 \%$ confluency. Monolayers were then covered with 5\% mucin MUC2 or MUC5b, and the ability of bacteria to enter the epithelial cells was investigated as previously reported $[8,9]$. In brief, monolayers were incubated with either M. avium 104 or $P$. aeruginosa, $E$. coli, S. aureus, and L. monocytogenes for 1 hour, the supernatant was removed, and the plate was washed with HBSS three times. The epithelial cell monolayer was than incubated at $37^{\circ} \mathrm{C}$ for 4 hours. Following the incubation, monolayers were lysed as previously described $[8,9]$, and the lysate was serially diluted and plated into $7 \mathrm{H} 10$ agar plates (Figure 1).

In some of the assays, bacteria were treated with $4 \mu \mathrm{g} / \mathrm{ml}$ of polymyxin B (antimicrobial peptide surrogate) alone or incubated with polymyxin B MUC2 mucin on a HEp-2 cell monolayer.

2.6. Statistical Analysis. The data were analyzed using GraphPad Prism. Student's $t$-test and ANOVA were used to determine the significance of the comparisons.

\section{Results}

3.1. M. avium Mucin Adhesion. To determine whether $M$. avium strains can bind to the mucin that comprises the intestinal mucosa, bacteria were incubated using an inoculum of approximately $10^{8} \mathrm{CFU} / \mathrm{ml}$ bacteria on the MUC2 mucin-covered tissue culture plate. After the incubation, each well in the mucin-covered plate was washed three times at room temperature with PBS. The bacteria removed was plated and counted as the nonadhered bacteria. A similar assay was carried out using mucin MUC5b.

Pseudomonas aeruginosa PD0300, a mucA22 mutant of PAO1, was used as a positive control for this assay as it binds to various mucins, including the porcine stomach mucin used as previously established [21, 22]. With the basic protocol, PD0300 showed a significant amount of binding to the mucin/agar compared with agar, whereas M. avium 104 showed little to no significant amount of binding to the mucin/agar compared with agar.
To determine whether binding to mucins was related to the pathogenic bacterium being Gram positive or Gram negative, Listeria monocytogenes, Staphylococcus aureus 25923, Escherichia coli CFT073, and Mycobacterium avium strains 104 and 3388 were assayed for mucin MUC2 binding. Gram-negative bacterial species (E. coli and $P$. aeruginosa) and Listeria monocytogenes showed a statistically significant greater percent of binding to the mucin/ agar compared to agar, and both $S$. aureus and $M$. avium showed little to no significant binding to the mucin/agar compared to agar (Table 1). In all M. avium species assayed, there was no significant difference seen in binding to the mucin/agar compared to agar. The results obtained regarding the binding to the MUC5b mucin were very similar (Table 2).

\subsection{Effect of Environmental Condition on Binding to Mucin.} To further investigate the possible factors affecting the binding of M. avium 104 to the MUC2 mucin, a number of pretreatments were performed prior to the binding assay basic protocol. Pretreatments of bacteria with $\mathrm{DI} \mathrm{H}_{2} \mathrm{O}$ and $\mathrm{HCl}\left(\mathrm{pH} 3.4\right.$ and $\mathrm{H}_{2} \mathrm{O}$ ) were done directly prior to the assay; M. avium strains (104 and 3388) were incubated in water $(12 \mathrm{~h})$ or acid with a $\mathrm{pH}$ of $4(2 \mathrm{~h})$, and the ability to bind to the mucin was examined. As shown in Table 3, environmental condition incubation had no effect on the binding to the mucin.

\subsection{Adherence to Mucosal Epithelial Cell}

M. avium does not express a flagellum, and therefore does not move quickly through the mucus layer. To determine whether a layer of mucus would interfere with the ability of M. avium to bind and invade mucosal epithelial cells, invasion of HEp-2 and A549 alveolar epithelial cells was evaluated in the presence or absence of a MUC2 mucin layer. As shown in Table 4, although the mucin interferes with the binding and invasion of host cells by $E$. coli, it had no effect on the invasion of the mucosal cells by two different strains of M. avium.

3.4. Treatment of M. avium with Tween-20 or Trypsin. The ability to prevent the binding to the mucin can be related to the cell wall of $M$. avium. To investigate the role of the bacterial surface, we treated $M$. avium with 5\% Tween-20 and $50 \%$ trypsin, prior to determination of binding to the 
TABLE 1: Binding of bacteria to the MUC2 mucin.

\begin{tabular}{lccc}
\hline Bacteria & \multicolumn{3}{c}{ Adherence to MUC2 mucin at different } \\
& $0.1(\%)$ & $1(\%)$ & $5(\%)$ \\
\hline M. avium 104 & 0 & 0 & 0 \\
M. avium 3388 & 0 & 0 & 0 \\
E. coli & $49.5 \pm 0.6^{*}$ & $51.0 \pm 1.2^{*}$ & $44.3 \pm 0.8^{*}$ \\
P. aeruginosa & $94.1 \pm 0.4^{*}$ & $92.6 \pm 0.5^{*}$ & $97.3 \pm 0.3^{*}$ \\
S. aureus & 0 & 0 & 0 \\
L. monocytogenes & $95.0 \pm 0.2^{*}$ & $96.2 \pm 0.4^{*}$ & $97.5 \pm 0.2^{*}$ \\
\hline
\end{tabular}

Bacteria were prepared as described in Section 2. Agar/mucin and mucin control were used. The numbers represent the subtraction of adherence from the background (agar alone). The adherence of E. coli, P. aeruginosa, and $L$. monocytogenes was statistically significant compared to that of $M$. avium and S. aureus $(p<0.05)$.

TABle 2: Binding of bacteria to mucin MUC5b.

\begin{tabular}{lcc}
\hline Bacteria & \multicolumn{2}{c}{$\begin{array}{c}\text { Adherence to mucin MUC5b } \\
\text { concentration (\%) }\end{array}$} \\
& $1(\%)$ & $5(\%)$ \\
\hline M. avium 104 & 0 & 0 \\
M. avium 3388 & 0 & 0 \\
E. coli & $59.0 \pm 0.9^{*}$ & $67.1 \pm 1.6^{*}$ \\
P. aeruginosa & $92.4 \pm 0.3^{*}$ & $93.8 \pm 0.4^{*}$ \\
L. monocytogenes & $94.3 \pm 0.3^{*}$ & $95.1 \pm 0.3^{*}$ \\
S. aureus & 0 & 0 \\
\hline
\end{tabular}

Bacteria were prepared as described in Section 2. Agar mucin and agar control were used. The numbers represent the subtraction of adherence from the background (agar alone). The adherence of E. coli, L. monocytogenes, and $P$. aeruginosa was significant when compared to that of $M$. avium and S. aureus $(p<0.05)$.

TABLE 3: Effect of environmental conditions on the binding to mucin MUC2.

\begin{tabular}{lccc}
\hline Bacteria & \multicolumn{3}{c}{ Binding to mucin (\%) } \\
& $\mathrm{H}_{2} 0(\%)$ & Acid $\mathrm{pH}(\%)$ & 7H9 (\%) \\
\hline M. avium 104 & 0 & 0 & 0 \\
M. avium 3388 & 0 & 0 & 0 \\
\hline
\end{tabular}

M. avium (strains 104 and 3388) was incubated in $\mathrm{H}_{2} 0$ (overnight) or HBSS $\mathrm{pH} 4.0$ (for $2 \mathrm{~h}$ ); the number of bacteria was adjusted to $10^{5}$ organisms, and binding was performed to agar/mucin. M. avium collected in the 7H9 broth was used as a control.

mucin. Both treatments significantly increased the binding of $M$. avium to the mucin, suggesting that the pathogen is surrounded by a cell wall that prevents the binding to the mucin and removal of the mucosal surface by a mechanical mechanism (Table 5).

The indication from the previous results suggests that the bacterium cell contents are very likely involved in the ability to prevent interaction with the mucin. To confirm the role of the bacterial surface on the binding to the mucin, we employed the M. avium 4B2 mutant (mutation on the gene $p s t B$, a synthase part of the glycopepdolipid synthesis pathway, 34) in the mucin binding assay. The results show that the M. avium 4B2 mutant can bind to the mucin, in contrast to the wild-type bacteria (Table 6).
TABLE 4: Invasion of epithelial cells by M. avium and E. coli in presence or absence of MUC2.

\begin{tabular}{lcccc}
\hline \multirow{2}{*}{ Bacteria } & \multicolumn{4}{c}{ Invasion (\%) } \\
& None & $5 \%$ mucin & None & $5 \%$ mucin \\
\hline M. avium 104 & $7.2 \pm 0.3$ & $7.0 \pm 0.5$ & $8.6 \pm 0.4$ & $8.3 \pm 0.3$ \\
M. avium 3388 & $6.8 \pm 0.4$ & $6.6 \pm 0.5$ & $7.9 \pm 2$ & $7.6 \pm 0.7$ \\
E. coli & $27 \pm 5$ & $16.7 \pm 3\left({ }^{*}\right)$ & $20 \pm 0.9$ & $11.0 \pm 0.4\left(^{*}\right)$ \\
\hline
\end{tabular}

$\left(^{*}\right) p<0.05$ compared with the invasion without the presence of mucin. Bacteria were prepared as described in Section 2. Epithelial cells, $90 \%$ confluent, were covered with $5 \%$ mucin, and bacterial invasion was determined by incubating bacteria with culture cells (with or without mucin) and quantifying the number of intracellular bacteria (percent of invasion). Initial inoculum of $2 \times 10^{5}$ for M. avium $104,2.4 \times 10^{5}$ for M. avium 3388, and $3.3 \times 10^{5}$ for $E$. coli was used.

TABLE 5: Effect of Tween-20 and trypsin treatment on the binding to the MUC2 mucin.

\begin{tabular}{lccc}
\hline \multirow{2}{*}{ Bacteria } & \multicolumn{3}{c}{$\%$ binding to MUC2 mucin } \\
& Control (7H9) & Tween-20 & Trypsin \\
\hline M. avium 104 & 0 & $47 \pm 6^{*}$ & $38.1 \pm 4^{*}$ \\
M. avium 3388 & 0 & $54 \pm 6^{*}$ & $48.4 \pm 5^{*}$ \\
\hline$p<0.05$ compared to $M$. avium incubated in the $7 \mathrm{H} 9$ broth. $M$. avium \\
strains were treated with Tween-20 or trypsin for $3 \mathrm{~h}$ at $37^{\circ} \mathrm{C}$, washed, and \\
incubated with 5\% MUC2 mucin. The numbers represent the percent of \\
binding to the mucin.
\end{tabular}

3.5. Effect of Polymyxin B on M. avium and Invasion of HEp-2 Cells. To evaluate whether polymyxin $B$, a surrogate for the antimicrobial peptide, had an antibacterial effect on a system containing mucosal cells and MUC2 mucin, both M. avium 104 and MAH mutant $4 \mathrm{~B} 2$ were incubated with $4 \mu \mathrm{g}$ of polymyxin $\mathrm{B}$ for 4 hours, and the number of bacteria was quantified, showing killing activity against MAH 4B2, but not $M$. avium 104. When MAH 4B2 was added to a HEp-2 monolayer covered with the mucin MUC2 in the presence of polymyxin B, the ability of the bacterium to enter the epithelial cell was significantly decreased compared with the wild-type M. avium 104 and with monolayer not containing polymyxin B (Table 7).

\section{Discussion}

The externally connected human host mucosal surface contains a layer or two of mucin, a glycoprotein intended to serve as a barrier to pathogens and other antigens $[10,11]$ and as a continuous cleaning mechanism for inhaled and ingested particles or microbes. Pathologic processes that impact the amount or fluidity of the mucin layer are associated with increase in the infections. Commonly observed conditions are chronic pulmonary diseases such as bronchiectasis, emphysema, and cystic fibrosis [23]. Those conditions course with the decrease in the mechanical defense of the lung mucosa, as well as the same for the intestinal mucosa.

Upon infection, cells producing mucin (mainly goblet cells) undergo hyperplasia and increased mucus secretion, 
TABLe 6: M. avium 104, A5, and 4B2 mutant binding to the mucin.

\begin{tabular}{lcc}
\hline Bacteria & \% bacteria to MUC2 mucin & Binding \\
\hline M. avium 104 & 0 & \% bacteria to MUC5b mucin \\
M. avium A5 & 0 & 0 \\
M. avium 4B2 & $32 \pm 3^{*}$ & 0 \\
\hline
\end{tabular}

Results demonstrated that the cell wall-associate mutant has increased ability to interact with MUC2 and MUC5b mucins. ${ }^{*} p<0.05$ compared to the binding of the wild-type bacteria. M. avium $4 \mathrm{~B} 2$ has been described previously, and it is deficient in colonization of the intestinal and respiratory mucosa. The mutant and wild-type were inhabited in a surface covered by MUC2 or MUC5b.

TABLE 7: Effect of polymyxin B on the invasion of HEp-2 cells in presence of the MUC2 mucin.

\begin{tabular}{lccc}
\hline Bacteria & Polymyxin B & Invasion HEp-2 & Invasion HEp-2+ polymyxin B \\
\hline M. avium 104 & $4.5 \pm 0.5 \times 105$ & $8.3 \pm 0.4 \times 104$ & $8.0 \pm 0.5 \times 104$ \\
MAH 4B2 & $2.5 \pm 0.6 \times 103^{*}$ & $3.2 \pm 0.5 \times 103^{*}$ & $6.4 \pm 0.4 \times 102^{* \% \&}$ \\
\hline
\end{tabular}

Inoculum was $4.5 \times 10^{5}$ for $M$. avium and $3 \times 10^{5}$ for MAH $4 \mathrm{~B} 2$. Bacteria were incubated with polymyxin for 4 hours, and the diluted and plated bacteria were placed on the monolayers with or without polymyxin for $2 \mathrm{~h}$; then extracellular bacteria were removed, and HEp-2 cells were lysed. The lysate was plated for quantification of colony forming units. ${ }^{*} p<0.5$ for the comparison between $M$. avium 104 and $4 \mathrm{~B} 2 . \% \& p<0.5$ for the comparison between MAH $4 \mathrm{~B} 2$ invasion of HEp-2 cells with and without polymyxin B.

proving protection against many different pathogens [24]. The layer of the mucin also participates in the tissue-related immune response, by incorporating antimicrobial peptides, enzymes such as lysozyme, and antibodies [11]. Recent publication also identified the innate lymphocytic cells (ILC 2 ) as an important trigger for mucin production in the sites connected with intestinal helminths [25].

Our investigation looked at the role of both intestinal and respiratory mucus in the mucosal defense against a nontuberculous mycobacteria, $M$. avium. The findings that the M. avium does not bind to either mucins (MUC2 and MUC5b), in contrast to $E$. coli and P. aeruginosa, suggest that $M$. avium crosses the mucin layer easily, explaining how the bacterium can attach to the mucosal surface without expressing a fimbria or long pili, which have been associated with the ability of another pathogen, $P$. aeruginosa, to bind and invade the lung airway mucosa [16].

Mucin protects against infection. A study reported by Bergstrom et al. and colleagues [14] investigating the role of pathogenic bacteria in lethal infectious colitis supports the concept that the mucin serves as a barrier to bacteria, preventing them from interacting with the mucosa surface. In fact, the work by Hashein et al. and colleagues [26] demonstrated that MUC2 deficiency resulted in increased susceptibility of mice to intestinal nematodes.

Pathogens interact with the mucus in different fashions. A common motility factor, flagella, is utilized by pathogenic bacteria such as Vibrio cholera to migrate through the mucus barrier. Similarly, another intestinal pathogen, Salmonella, appears to anchor itself to the mucus layer, using adhesions to promote colonization $[27,28]$.

Recent investigation showed that the airway mucus restricts Neisseria meningitides access to the nasopharyngeal epithelial cells, protecting the respiratory mucosa from the bacterium and the associated inflammatory response [29]. In that specific study, the presence of mucus on the cell surface prevented the inflammatory response from the epithelial cells, which may alter the interaction of the bacteria with the mucosal cells. In the current study, we showed that the presence of mucus on the monolayer did not alter the ability of $M$. avium strains to interact with respiratory epithelial cells, again confirming that the barrier between the bacterium and the mucosal cells is not able to interfere with the binding.

M. avium is an environmental bacterium that many times infects the host through contact with environment sources [30]. Previous studies have shown that the passage of the bacterium in environmental conditions, for example, affect the physical properties of the bacterial surface, making it more resistant to the $\mathrm{pH}$ of the stomach [22]. Since M. avium is encountered in water, and to infect the intestinal mucosal epithelial cells, it needs to cross the acid environment of the stomach, we exposed the bacterium to both conditions and examined its ability to bind to the mucin, which showed that those conditions had an effect on the ability of M. avium to interact with the mucin. In fact, the lack of influence was not surprising, since binding to the mucin would leave no deleterious effect on the ability to bind to mucosal cells.

All the evidence collected suggest that the bacterial surface had a major impact on the interaction with the mucin. M. avium surface, like other mycobacteria, contains glycoproteins, lipids, and carbohydrates [26]. In fact, by treating the bacterium with either a detergent (Tween) or trypsin caused significant alteration in the content of the cell wall, with change in the ability to bind to the mucin. The same kind of observation has been described in other microorganisms in the interaction with mucosal structures. All the work performed with the mucin and bacteria indicate that the role of mucin is to protect the mucosal surface from pathogens, and M. avium has evolved a surface, probably changed in a manner, that it does not bind to either MUC2 or MUC5b. Using a $M$. avium mutant that has an altered glycolipid structure on the cell wall also increased the 
binding of the bacterium to the mucin. Because S. aureus was also incapable to bind to the mucin, we can assume that although the surface lipids may inhibit the binding of $M$. avium to the mucin, other structures also present on the $S$. aureus surface may be involved in the inhibition. Many other pathogens have been described interacting with the mucin, such as Campylobacter and helminths [31, 32], either by changing gene expression or promoting mucin induction of T cell-simulation cytokines.

The lack of efficacy of the mucin to create a barrier between the pathogen and the mucosal cells was demonstrated using two different epithelial cell lines, HEp-2 and A549. The observation adds to a previous finding that showed $M$. avium resistance to the action of antimicrobial peptides [33]. Taking in consideration that both the intestinal and respiratory mucosa are covered by two layers of mucin, crossing the top layer will put the bacteria in contact with the bottom layer, which is rich in antibacterial peptides. In fact, in our studies, incubation with a surrogate of antimicrobial peptides significantly impaired the ability of the bacterium to invade epithelial cells in a mucin-containing monolayer. Therefore, the capability to prevent the action of antimicrobial peptides complement the bacterial phenotype.

In summary, the study has shown that $M$. avium is capable of bypassing the action of mucin, a natural barrier to pathogens, and interacts with epithelial cells on the mucosa, despite of the absence of a flagellum. How M. avium acquired that pathogenic characteristic is unknown at this point. Future work will explore this aspect of the pathogen, probably by screening for mutants that bind to the mucin, such as the $4 \mathrm{~B} 2$ mutant in the work.

\section{Data Availability}

All the data included in the manuscript are available any time upon request.

\section{Conflicts of Interest}

The authors have no conflicts of interest to declare.

\section{Authors' Contributions}

JB performed experiments and wrote the paper. LEB performed experiments and edited the manuscript.

\section{Acknowledgments}

The authors thank the support for this research provided by the Microbiology Foundation of San Francisco. The authors are also grateful to Lynette Hawthorne and Amy Ma for the preparation of the manuscript. The authors are also in debt to Dr. Sasha Rose for the technical help.

\section{References}

[1] B. Kendall and K. Winthrop, "Update on the epidemiology of pulmonary nontuberculous mycobacterial infections," Seminars in Respiratory and Critical Care Medicine, vol. 34, no. 1, pp. 87-94, 2013.
[2] D. E. Griffith, T. Aksamit, B. A. Brown-Elliott et al., "An official ATS/IDSA statement: diagnosis, treatment, and prevention of nontuberculous mycobacterial diseases," American Journal of Respiratory and Critical Care Medicine, vol. 175, no. 4, pp. 367-416, 2007.

[3] S. M. Holland, "Novel defects in host defense evinced as nontuberculous mycobacterial and dimorphic mold infection: GATA2 deficiency, STAT1 mutations, anticytokine autoantibodies," Blood, vol. 118, no. 21, 2011.

[4] L. E. Bermudez and L. S. Young, "Factors affecting invasion of HT-29 and HEp-2 epithelial cells by organisms of the $M y$ cobacterium avium complex," Infection and Immunity, vol. 62, no. 5, pp. 2021-2026, 1994.

[5] L. E. Bermudez and F. J. Sangari, "Cellular and molecular mechanisms of internalization of mycobacteria by host cells," Microbes and Infection, vol. 3, no. 1, pp. 37-42, 2001.

[6] G. Carter, M. Wu, D. C. Drummond, and L. E. Bermudez, "Characterization of biofilm formation by clinical isolates of Mycobacterium avium," Journal of Medical Microbiology, vol. 52, no. 9, pp. 747-752, 2003.

[7] S. J. Rose, L. M. Babrak, and L. E. Bermudez, "Mycobacterium avium possesses extracellular DNA that contributes to biofilm formation, structural integrity, and tolerance to antibiotics," PLoS One, vol. 10, no. 5, Article ID e0128772, 2015.

[8] L. Babrak, L. Danelishvili, S. J. Rose, and L. E. Bermudez, "Microaggregate-associated protein involved in invasion of epithelial cells by Mycobacterium avium subsp. hominissuis," Virulence, vol. 6, no. 7, pp. 694-703, 2015.

[9] L. Babrak, L. Danelishvili, S. J. Rose, T. Kornberg, and L. E. Bermudez, "The environment of "Mycobacterium avium subsp. hominissuis" microaggregates induces synthesis of small proteins associated with efficient infection of respiratory epithelial cells," Infection and Immunity, vol. 83, no. 2, pp. 625-636, 2015.

[10] M. Caldara, R. S. Friedlander, N. L. Kavanaugh, J. Aizenberg, K. R. Foster, and K. Ribbeck, "Mucin biopolymers prevent bacterial aggregation by retaining cells in the free-swimming state," Current Biology, vol. 22, no. 24, pp. 2325-2330, 2012.

[11] M. E. V. Johansson, M. Phillipson, J. Petersson, A. Velcich, L. Holm, and G. C. Hansson, "The inner of the two Muc2 mucin-dependent mucus layers in colon is devoid of bacteria," Proceedings of the National Academy of Sciences, vol. 105, no. 39, pp. 15064-15069, 2008.

[12] C. Ridley and D. J. Thornton, "Mucins: the frontline defense of the lung," Biochemical Society Transactions, vol. 46, no. 5, 2018.

[13] J. F. Sicard, G. L. Bihan, P. Vogeleer, M. Jacques, and J. Harel, "Interactions of intestinal bacteria with components of intestinal mucus," Frontiers in Cellular and Infection Microbiology, vol. 7, 2017.

[14] K. S. B. Bergstrom, V. Kissoon-Singh, D. L. Gibson et al., "Muc2 protects against lethal infectious colitis by disassociating pathogenic and commensal bacteria from the colonic mucosa," PLoS Pathogens, vol. 6, no. 5, Article ID e1000902, 2010.

[15] A. T. Yeung, A. Parayno, and R. E. Hancock, "Mucin promotes rapid surface motility in Pseudomonas aeruginosa," mBio, vol. 3, no. 3, 2012.

[16] G. A. O'Toole and R. Kolter, "Flagellar and twitching motility are necessary for Pseudomonas aeruginosa biofilm development," Molecular Microbiology, vol. 30, no. 2, pp. 295-304, 1998.

[17] P. Kumar, Q. Luo, T. J. Vickers, A. Sheikh, W. G. Lewis, and J. M. Fleckenstein, "EatA, an immunogenic protective antigen 
of enterotoxigenic Escherichia coli, degrades intestinal mucin," Infection and Immunity, vol. 82, no. 2, pp. 500-508, 2014.

[18] J. L. Everman, N. R. Ziaie, J. Bechler, and L. E. Bermudez, "Establishing Caenorhabditis elegans as a model for Mycobacterium avium subspecies hominissuis infection and intestinal colonization," Biology Open, vol. 4, no. 10, pp. 1330-1335, 2015.

[19] Y. Yamazaki, L. Danelishvili, M. Wu, M. MacNab, and L. E. Bermudez, "Mycobacterium avium genes associated with the ability to form a biofilm," Applied and Environmental Microbiology, vol. 72, no. 1, pp. 819-825, 2006.

[20] Y. Yamazaki, L. Danelishvili, M. Wu et al., "The ability to form biofilm influences Mycobacterium avium invasion and translocation of bronchial epithelial cells," Cellular Microbiology, vol. 8, no. 5, pp. 806-814, 2016.

[21] K. Mathee, O. Ciofu, C. Sternberg et al., "Mucoid conversion of Pseudomonas aeruginos by hydrogen peroxide: a mechanism for virulence activation in the cystic fibrosis lung," Microbiology, vol. 145, no. 6, pp. 1349-1357, 1999.

[22] T. Bodmer, E. Miltner, and L. E. Bermudez, "Mycobacterium avium resists exposure to the acidic conditions of the stomach," FEMS Microbiology Letters, vol. 182, no. 1, pp. 45-49, 2000.

[23] W. J. Janssen, A. L. Stefanski, B. S. Bochner, and C. M. Evans, "Control of lung defence by mucins and macrophages: ancient defence mechanisms with modern functions," European Respiratory Journal, vol. 48, no. 4, pp. 1201-1214, 2016.

[24] J. V. Fahy and B. F. Dickey, "Airway mucus function and dysfunction," New England Journal of Medicine, vol. 363, no. 23, pp. 2233-2247, 2010.

[25] L. Campbell, M. R. Hepworth, J. Whittingham-Dowd et al., "ILC2s mediate systemic innate protection by priming mucus production at distal mucosal sites," Journal of Experimental Medicine, vol. 216, no. 12, pp. 2714-2723, 2019.

[26] S. Z. Hasnain, H. Wang, J. E. Ghia et al., "Mucin gene deficiency in mice impairs host resistance to an enteric parasitic infection," Gastroenterology, vol. 138, no. 5, 2010.

[27] D. Chessa, M. G. Winter, M. Jakomin, and A. J. Bäumler, "Salmonella enterica serotype typhimurium std fimbriae bind terminal $\alpha(1,2)$ fucose residues in the cecal mucosa," Molecular Microbiology, vol. 71, no. 4, pp. 864-875, 2009.

[28] S. R. Shames, S. D. Auweter, and B. B. Finlay, "Co-evolution and exploitation of host cell signaling pathways by bacterial pathogens," The International Journal of Biochemistry \& Cell Biology, vol. 41, no. 2, pp. 380-389, 2009.

[29] M. Audry, C. Robbe-Masselot, J.-P. Barnier et al., "Airway mucus restricts Neisseria meningitidis away from nasopharyngeal epithelial cells and protects the mucosa from inflammation," mSphere, vol. 4, no. 6, 2019.

[30] C. L. Tzou, M. A. Dirac, A. L. Becker et al., "Association between Mycobacterium avium complex pulmonary disease and mycobacteria in home water and soil," Annals of the American Thoracic Society, vol. 17, no. 1, pp. 57-62, 2020.

[31] S. Z. Hasnain, C. M. Evans, M. Roy et al., "Muc5ac: a critical component mediating the rejection of enteric nematodes," The Journal of Experimental Medicine, vol. 208, no. 5, pp. 893-900, 2011.

[32] Q. V. Tu, M. A. McGuckin, and G. L. Mendz, "Campylobacter jejuni response to human mucin MUC2: modulation of colonization and pathogenicity determinants," Journal of Medical Microbiology, vol. 57, no. 7, pp. 795-802, 2008.

[33] N. Motamedi, L. Danelishvili, and L. E. Bermudez, "Identification of Mycobacterium avium genes associated with resistance to host antimicrobial peptides," Journal of Medical Microbiology, vol. 63, no. 7, pp. 923-930, 2014.

[34] S. M. Fleiszig, T. S. Zaidi, R. Ramphal, and G. B. Pier, "Modulation of Pseudomonas aeruginosa adherence to the corneal surface by mucus," Infection and Immunity, vol. 62, no. 5, pp. 1799-1804, 1994.

[35] L. Chiaradia, C. Lefebvre, J. Parra et al., "Dissecting the mycobacterial cell envelope and defining the composition of the native mycomembrane," Scientific Reports, vol. 7, no. 1, Article ID 12807, 2017. 